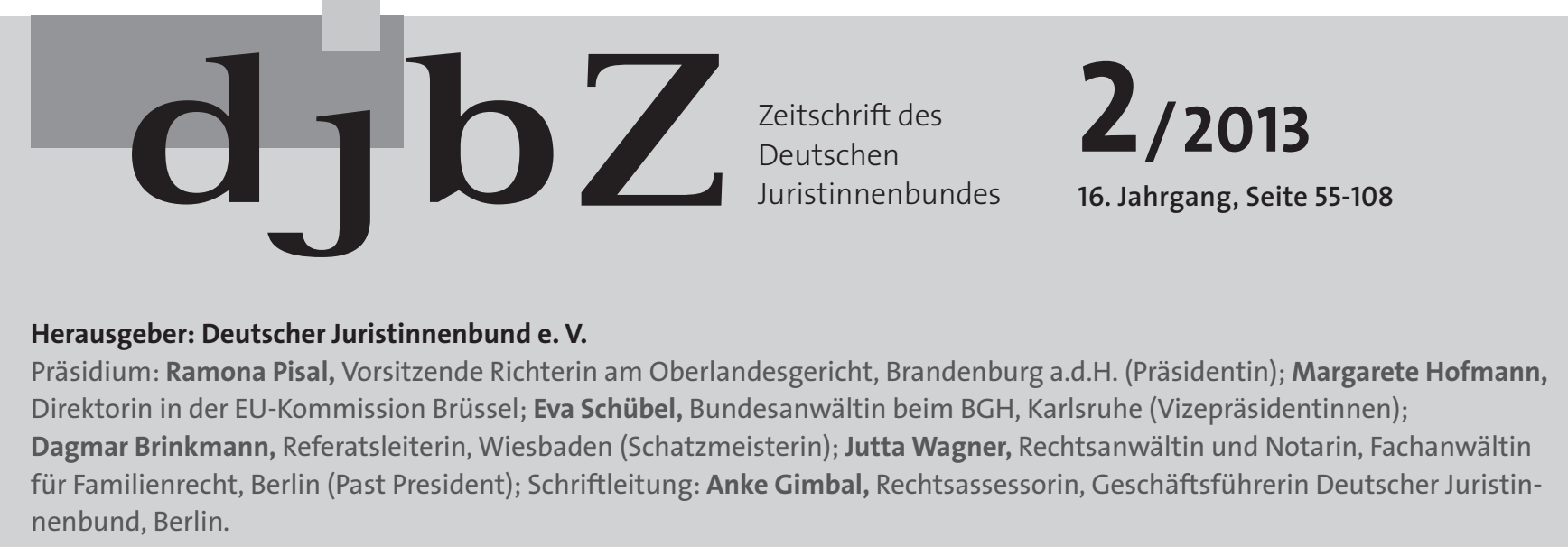

\title{
Wir haben die Wahl - Forderungskatalog des djb
}

Der Deutsche Juristinnenbund ( $\mathrm{djb}$ ) fordert von der neuen Bundesregierung vor allem eine konsistente Gleichstellungspolitik. Sie muss sicherstellen, dass Frauen nicht die Folgen von Gesetzen zu tragen haben, die auf unterschiedlichen Familienbildern beruhen - wie im Falle von Unterhaltsrecht und Einkommensteuerrecht.

Frauen müssen die realistische Chance haben, gleichberechtigt für die eigenständige Sicherung ihrer Existenz zu sorgen, im gesamten Lebensverlauf. Dafür sind unter anderem gleiche Karrierechancen und Entgeltgleichheit unerlässlich.

Unser Forderungskatalog umfasst zentrale frauenpolitisch relevante Politikfelder wie zum Beispiel Gleichstellung in der Privatwirtschaft, im öffentlichen Dienst und im Vergaberecht,
Entgeltgleichheit, Vereinbarkeit von privater Sorge und Familie mit der Erwerbsarbeit, eheliches Güterrecht, Unterhaltsrecht, Adoptionsrecht, vertrauliche Geburt, Erbrecht, Menschenhandel, Sexualdelikte, Schutz vor häuslicher Gewalt, Opferschutz, SGB II, Alterssicherung, Krankenversicherung, Betreuungs- und Elterngeld, Steuerpolitik, Migrationspolitik, Staatsbürgerschaft, Europapolitik, Mutterschutz und vieles mehr.

Wir bitten unsere Mitglieder und appellieren an alle, sich aktiv in den kommenden Bundestagswahlkampf einzubringen, die Kandidat/inn/en vor Ort anhand dieser Forderungen zu befragen, die Antworten zu vergleichen und in ihre Entscheidung zur Wahl einfließen zu lassen.

\section{Kommission Arbeits-, Gleichstellungs- und Wirtschaftsrecht ${ }^{1}$}

Auch über 50 Jahre nach Gründung der Bundesrepublik und Geltung des Grundgesetzes sind Frauen in der Erwerbsarbeit noch nicht gleichberechtigt: Sie verdienen im Durchschnitt 23 Prozent weniger als Männer. Sie sind in Führungspositionen so selten vertreten, dass von einer gläsernen Decke gesprochen werden muss. Die ungleiche Repräsentation der Geschlechter auf allen Ebenen und in allen beruflichen Bereichen zeigt, dass darüber hinaus auch gläserne Wände bestehen, in denen Arbeitnehmerinnen und Arbeitnehmer eingeschlossen sind. Altersarmut ist nicht zufällig vor allem ein Problem weiblicher Lebensläufe. Sie ist der Tatsache geschuldet, dass sich typisch weibliche Lebensläufe durch Diskontinuitäten, Teilzeitarbeit, Beschäftigung im Niedriglohnsektor und im Vergleich mit Männern schlechtere Bezahlung auszeichnen.

Vor diesem Hintergrund hält der djb im Arbeits-, Wirtschafts- und Gleichstellungsrecht auf gesetzlicher Ebene insbesondere die folgenden Maßnahmen für erforderlich:

\section{A. Gleichstellung in der Privatwirtschaft}

Ein umfassendes „Gesetz zur Gleichstellung der Geschlechter in der Privatwirtschaft“ steht seit Langem aus. Die „Freiwillige Vereinbarung zwischen der Bundesregierung und den Spitzenverbänden der deutschen Wirtschaft" von 2001 hat sich als unwirksam erwiesen. ${ }^{2}$

Es wird Aufgabe der nächsten Bundesregierung sein, den Handlungsauftrag des Artikels 3 Absatz 2 Satz 2 GG endlich durch ein solches Gesetz umzusetzen. Es sollte zahlreiche Einzelmaßnahmen, die in den letzten Jahren teilweise isoliert begonnen wurden, in einem Gesamtkonzept verbinden.

1 Redaktion: Prof. Dr. Eva Kocher, Europa-Universität Viadrina, Frankfurt (Oder).

2 Vgl. die djb-Pressemitteilungen und-Stellungnahmen zu den Bilanzen seit 2001, exemplarisch „Stagnation auf niedrigem Niveau“ v. 26.6.2008 u. zuletzt Bericht „Aktionärinnen fordern Gleichberechtigung: Das Dogma von Qualifikation und Leistung“ in djbZ 3/2012, S. 121. 
Die einzelnen Elemente eines „Gesetzes zur Gleichstellung in der Privatwirtschaft" sollten sein:

- ein verbindliches Verfahren zur Herstellung von Entgeltgleichheit sowie Einführung eines allgemeinen Mindestlohns,

- die Quotierung von Führungspositionen und eine Reform des Allgemeinen Gleichbehandlungsgesetzes (AGG),

- gesetzliche Maßnahmen zur Vereinbarkeit von privater Sorge und Familie mit Erwerbsarbeit für alle Personen; dazu gehört auch die vollständige soziale Sicherung jeglicher abhängiger Arbeit und damit die Abschaffung der Sozialversicherungsfreiheit von Minijobs,

- ein verbindliches Verfahren zur Findung von proaktiven betrieblichen Gleichstellungsmaßnahmen mit Koppelung öffentlicher Aufträge.

\section{Dies bedeutet im Einzelnen:}

\section{Entgeltgleichheit}

\section{a) Verbindliches Verfahren}

Der Rechtsanspruch auf Entgeltgleichheit ist nicht verwirklicht. Frauen und Männer werden bei gleicher oder gleichwertiger Arbeit oft nicht gleich bezahlt. Nicht selten wird dies damit begründet, dass Frauen schlechter verhandelt hätten - ein rechtlich nicht haltbares Argument. Auch Tarifverträge lassen verschiedenste Mechanismen mittelbarer Entgeltdiskriminierung erkennen: So wird zum Beispiel die Tätigkeit im Verkauf mit dreijähriger Berufsausbildung im Einzelhandel schlechter entlohnt als eine Lagerarbeitertätigkeit ohne Ausbildung. Ein sachlicher Grund für diese Differenz ist nicht erkennbar, ursächlich hierfür dürften tradierte Entgeltstrukturen und gesellschaftliche Geringbewertung von sogenannter „weiblicher“ Arbeit sein. Es werden vielfach psychosoziale Anforderungen der Kommunikation, der Kooperation oder des Einfühlungsvermögens verlangt, bei der Bezahlung jedoch nicht honoriert. Verantwortung wird oft nur im Hinblick auf Finanzen oder Führung und nicht im Hinblick auf Menschen bewertet. Physische Anforderungen und Belastungen werden überwiegend bei gewerblichen Tätigkeiten anerkannt und vergütet, nicht aber bei Dienstleistungstätigkeiten wie etwa in der Altenpflege.

Statt die Entgeltlücke „schön“ zu rechnen ${ }^{3}$, sollten Wirtschaft, Politik und Wissenschaft ihre Energie darauf verwenden, effektive Maßnahmen zur Beseitigung von Entgeltdiskriminierung zu ergreifen. Zahlreiche Pilotprojekte haben gezeigt, wie betriebliche und überbetriebliche Verfahren zur Herstellung von Entgeltgleichheit funktionieren könnten. Die Einbeziehung der Tarifparteien ist dabei wichtig. Eine gesetzliche Verpflichtung zur Durchführung solcher Maßnahmen ist erforderlich, denn Unternehmen nutzen bislang weder ihre Informationsnoch ihre Handlungsmöglichkeiten in nennenswertem Umfang.

\section{Der djb fordert}

- eine gesetzliche Verpflichtung zur geschlechtsdifferenzierten Entgeltanalyse im Betrieb,
- eine gesetzliche und fristgebundene Verpflichtung, unter Beteiligung betrieblicher Akteur/inn/e/n, der Tarifparteien und externer Sachverständiger geschlechtergerechte Entgeltregelungen einzuführen,

- spürbare Sanktionen bei Verstößen oder Untätigbleiben.

- Fehlverhalten oder Unterlassen der Arbeitgeber müssen mittels Verbandsklage oder durch die Antidiskriminierungsstelle des Bundes rechtsförmig angreifbar sein. Diesbezügliche Versäumnisse im AGG (siehe unten) dürfen hier nicht wiederholt werden.

\section{b) Mindestlohn}

Eine zentrale Rolle bei der Gewährleistung existenzsichernder Erwerbsarbeit kommt Mindestlohnregelungen zu. Gerade für Frauen wären sie ein wichtiges Instrument der Existenz- und Alterssicherung. Denn tarifautonome Entgeltfestsetzung kann nur dort die sozialen Funktionen eines Mindestentgelts erfüllen, wo es ausreichende gewerkschaftliche Verhandlungsstärke gibt - dies ist jedoch in Bereichen, in denen typischerweise Frauen arbeiten, häufig gerade nicht der Fall. Der gesetzliche Mindestlohn ist ein erstes und wichtiges Instrument, um die Entgeltlücke zwischen den Geschlechtern zu verkleinern.

\section{Der djb fordert}

- einen gesetzlichen Mindestlohn in angemessener Höhe.

\section{Rechte gegen gläserne Decken und gläserne Wände}

\section{a) Quotierung von Führungspositionen}

Frauen sind in der Bundesrepublik sehr gut ausgebildet. Dennoch kann auch in Zukunft nicht darauf vertraut werden, dass der demografische Wandel ohne weiteres dazu führen wird, Frauen endlich gleichberechtigte Verantwortung in Führungspositionen zu ermöglichen. Diskriminierungen werden oftmals zu Unrecht für wirtschaftlich effizient gehalten. Tatsächlich sind Unternehmen mit divers zusammengesetzten Belegschaften ökonomisch erfolgreicher. Solange traditionelle Geschlechterstereotypen über die Aufstiegsmöglichkeiten entscheiden, bleibt Frauen der Zugang zu Führungspositionen verwehrt. Eine gesetzliche Mindestquote für Frauen gebietet sich schon aufgrund Artikel 3 Absatz 2 Satz 2 GG. Die Präsenz von Frauen in Führungspositionen ist ein Schlüssel zu wirtschaftlicher Stabilität und Wachstum. Auch die EU-Kommission tritt deshalb für entsprechende Regelungen ein.

Angesichts der aktuellen Zahlen im Aufsichtsrat, Vorstand, in den Ausschüssen des Aufsichtsrates und den Führungspositionen unterhalb des Vorstandes besteht insbesondere an diesen Stellen dringender Handlungsbedarf. Rund 80 Prozent aller Aufsichtsratspositionen der DAX30-Unternehmen waren 2012 mit Männern besetzt, 19,4 Prozent mit Frauen, davon jedoch nur 7,6 Prozent auf Anteilseignerseite. In den Nominierungsausschüssen für die Aufsichtsratspo-

3 So aber Schäfer, Holger/Schmidt, Jörg/Stettes, Oliver, Beschäftigungsperspektiven von Frauen, Köln 2013. 
sitionen saßen 2012 nur sieben Frauen. In den Personalausschüssen betreffend die Bestellung der Vorstände saßen 2012 nur fünf Frauen, die alle von Arbeitnehmerseite gestellt wurden. 92 Prozent der Vorstandspositionen der DAX30Unternehmen sind mit Männern besetzt. In den Geschäftsberichten wird Beschäftigung nur vereinzelt nach Geschlecht aufgeschlüsselt, nur ein einziges DAX30-Unternehmen legt seinen Frauenanteil auf allen vier Führungsebenen unterhalb des Vorstandes offen. ${ }^{4}$ Die politische Gestaltungsmacht darf nicht weiterhin auf Appelle an Freiwilligkeiten, Flexiquoten und ähnliches begrenzt werden.

\section{Der djb fordert}

- eine gesetzliche Frauenquote von 40 Prozent für Aufsichtsratspositionen, für die Besetzung der Ausschüsse des Aufsichtsrates und für Vorstandspositionen,

- eine Ergänzung von $\$ 289$ Absatz 3 HGB (Lagebericht) um die Klarstellung, dass der Anteil der Geschlechter an der Gesamtzahl der Arbeitnehmer/innen und Organmitglieder auf den verschiedenen Hierarchieebenen, insbesondere im unteren, mittleren und Top-Management des Unternehmens, für die Lage der Gesellschaft immer von Bedeutung ist,

- eine gesetzliche Verpflichtung, Auswahlkriterien und Auswahlverfahren für Führungspositionen transparent zu machen und jährlich Angaben über den Anteil der Frauen in der Belegschaft, den Organen und in den Führungsebenen zu veröffentlichen,

- spürbare Sanktionen bei Verstößen.

\section{b) Reform des AGG}

Der Erlass des AGG im Jahre 2006 war mit vielen Hoffnungen verbunden, die sich nicht erfüllt haben. Das Gesetz konnte nur vereinzelt Veränderungen bewirken. Auf die neuen gesetzlichen Diskriminierungsverbote reagieren die Betriebe bestenfalls passiv. Strukturell hat sich wenig verändert, da die Betriebe keinem besonderen Handlungsdruck unterworfen wurden: Es fehlt an Rechtsbindung und Sanktionsdruck.

Ein Erkennen von systematischen Diskriminierungsmustern und ihre Beseitigung über den Einzelfall hinaus kann nicht nur durch Individualklagen bewirkt werden. Eine echte Verbandsklage könnte es unabhängigen Verbänden und Antidiskriminierungsstellen ermöglichen, Ungleichheiten und Diskriminierungsmuster, die repräsentativ für gesellschaftliche (Fehl-)Entwicklungen stehen, gezielt und aus eigenem Antrieb anzugreifen und hier spezialisierte Expertise einzubringen. Daneben muss die Möglichkeit zur Individualklage verbessert werden, um Klagen gegen Diskriminierungen und damit auch Anstöße zu Änderungsprozessen „von unten“ effektiv zu ermöglichen. Die Entschädigungsansprüche sind hier in ihrer Höhe aktuell so niedrig und die Auslegung der Beweislastregelung so streng, dass sie Betroffene entmutigen, ihre Ansprüche durchzusetzen. Den Gerichten fehlt es an klaren Hinweisen des Gesetzgebers, welche Tatsachen beispielhaft darauf schließen lassen, dass ihnen eine bestimmte (benachteiligende) Unternehmens-/Personalpolitik oder Motivation zugrunde liegt.
Das Bundesarbeitsgericht hat mit seiner Rechtsprechung zur Wertung von statistischen Indizien für systematische Diskriminierung als Ergebnis von „echtem Zufall“ ${ }^{5}$ bestehende Beweiserleichterung hier noch wirkungsloser gemacht.

\section{Der djb fordert:}

- Die Verbandsklage für Gewerkschaften und Antidiskriminierungsverbände,

v eine Erhöhung der Entschädigungsansprüche,

- gesetzliche Auslegungsvorgaben für die Beweislastregelung, die klarstellen, dass (und welche) statistische und andere Indizien die Beweislast auf die Arbeitgeberseite verlagern,

- flankierende Auskunftsansprüche für potentiell Benachteiligte.

\section{Vereinbarkeit von privater Sorge und Familie mit der Erwerbsarbeit}

\section{a) Arbeitszeiten, Elternzeiten, Pflegezeiten}

Inzwischen setzen viele Unternehmen auf eine Flexibilisierung bei Arbeitszeiten zum Beispiel durch variable Arbeitszeiten, Teilzeit und Arbeitszeitkonten. Dies funktioniert in der Regel gut, soweit diese Instrumente den betrieblichen Bedürfnissen dienen. Private Belange der Beschäftigten werden im Rahmen der bestehenden betrieblichen Rahmenbedingungen jedoch meist nur unzureichend berücksichtigt. Hindernd wirken oft tradierte Vorstellungen (z.B. „Führungsaufgaben lassen sich nicht in Teilzeit erbringen “). Private Zeitbedarfe wird die betriebliche Praxis auch künftig auf breiter Ebene nur berücksichtigen, soweit die betrieblichen Rahmenbedingungen dies möglich machen. Beschäftigte müssen deshalb durchsetzbare Rechte erhalten, mit denen familiäre Zeitbedarfe und private Zeitautonomie eingefordert und eine bestehende betriebliche Praxis auf Machbarkeiten und Flexibilisierung überprüft werden kann. Im Anschluss an entsprechende Gerichtsentscheidungen $^{6}$ ist zum Beispiel der Anspruch der Beschäftigten auf Berücksichtigung von familiären Anliegen bei der Festlegung der Lage der Arbeitszeit gesetzlich festzuschreiben.

Das Teilzeit- und Befristungsgesetz (TzBfG) war ein wichtiger Einstieg, ist aber einen Anspruch auf Arbeitszeitverlängerung nach der Teilzeit schuldig geblieben. Es geht nicht nur um die Ermöglichung von Teilzeitarbeit, sondern auch um mehr Zeitsouveränität auf Vollzeitarbeitsplätzen und den „Wiedereinstieg “ nach Teilzeiten und Fürsorgezeiten.

Für die Elternzeit sind die Möglichkeiten für eine gleichberechtigte Aufteilung der privaten Sorgearbeit zu verbessern. Dies betrifft insbesondere die Möglichkeit, dass beide Elternteile gleichzeitig Elternzeit nehmen, insbesondere in Teilzeit.

4 So die Daten der Studie des Bundesministeriums für Familie, Senioren, Frauen und Jugend (Hg.), „Aktionärinnen fordern Gleichberechtigung - 2012“, Berlin 2012.

5 BAG, NZA 2011, S. 99.

6 BAG v. 23.9.2004 - 6 AZR 567/03; LAG Rostock v. 26.11.20082 Sa $217 / 08$. 
Ein drängendes Problem für die Kontinuität weiblicher Erwerbsbiografien ist der „Wiedereinstieg“ bzw. die Rückkehr aus der Elternzeit. Aus Artikel 3 Absatz 2 und Artikel 6 Absatz 1 GG resultiert ein Schutzauftrag an den Gesetzgeber, eine Rückkehr in eine Beschäftigung und einen beruflichen Aufstieg (auch) nach der Kinderbetreuung zu ermöglichen. ${ }^{7}$ Hier bedarf es einer expliziten Regelung des Rechts, an den früheren Arbeitsplatz zurückzukehren, beziehungsweise, wenn das nicht möglich ist, einer gleichwertigen oder ähnlichen Tätigkeit zugewiesen zu werden. Bisher ist nur die Weiterbeschäftigung garantiert. Ein weiteres Recht auf Neuverteilung der Arbeitszeit beziehungsweise auf „Arbeitsarrangements“ in jeder Hinsicht ist auch durch die Elternurlaubsrichtlinie 2010/18/EU geboten.

Ausdrücklich betont werden muss, dass alle rechtspolitischen Maßnahmen nur erfolgreich sein können, wenn sowohl auf staatlicher als auch auf Unternehmensseite genügend Verantwortung übernommen und Geld verfügbar gemacht wird, um Fürsorge für Kinder und alte Menschen als Daseinsvorsorge für alle ausreichend und flexibel zur Verfügung zu stellen.

Völlig unzureichend geregelt ist bislang die Vereinbarkeit von Erwerbsarbeit und Pflege. Die Lösung des neuen Familienpflegezeitgesetzes, dass Beschäftigte sich eine längere Pflegezeit vorher und nachher selbst erarbeiten müssen, geht an der Pflegerealität vorbei und belastet praktisch einseitig die Frauen mit einem Verzicht auf Erwerbsarbeitszeit und Erwerbseinkommen - ganz abgesehen davon, dass ein gesetzlicher Anspruch auf entsprechende Arbeitszeitreduzierung fehlt. Entsprechend der Regelung zum Elterngeld ist auch in der Pflegezeit ein Recht auf Pflegezeit mit Pflegeunterstützungsgeld erforderlich.

\section{Der djb fordert,}

- dass der Rechtsanspruch auf Teilzeit auch für einen befristeten Zeitraum geltend gemacht werden kann,

- einen Rechtsanspruch von Teilzeitbeschäftigten, auf Vollzeit zu wechseln,

- eine Klarstellung des Rechtsanspruchs, die Arbeitszeitlage an familiäre Bedürfnisse anzupassen,

- die rechtliche Möglichkeit, gleichzeitig mit dem anderen Elternteil Elternzeit in Teilzeit zu nehmen, ohne den Anspruch dadurch gleich doppelt zu verbrauchen, zeitweilige Arbeitszeitarrangements nach der Elternzeit zu wählen und nach der Elternzeit auf den früheren oder einen gleichwertigen Arbeitsplatz zurückzukehren,

- Pflegezeit in gleicher Weise wie Elternzeit in sozial abgesicherter Form zu beanspruchen.

\section{b) Minijobs}

Bereits der Deutsche Juristentag von 2010 hat festgestellt, dass die Sozialversicherungsfreiheit von Minijobs die falschen Anreize setzt. Sie macht es insbesondere Frauen unmöglich, aus dem Niedriglohnsektor herauszukommen und schreibt Altersarmut fest.

\section{Der djb fordert}

- die Abschaffung der Sozialversicherungsfreiheit von Minijobs.

\section{Betrieblich angepasste positive Gleichstellungsmaßnahmen}

\section{a) Verbindliche Verfahren}

Für die Herstellung tatsächlicher Gleichberechtigung in den Betrieben gibt es keine Patentrezepte. Dennoch sind über die bereits erwähnten gesetzlichen Rechte hinaus positive Maßnahmen erforderlich, die an den jeweiligen Betrieb angepasst sind. Hierzu müssen die Entscheidungsträgerinnen und Entscheidungsträger in den Betrieben selbst in die Pflicht genommen werden. Es bedarf deshalb eines gesetzlichen Handlungsrahmens, der die Betriebe zur Planung, Durchführung und Kontrolle betrieblicher Gleichstellungspolitiken verpflichtet.

Vorbild für eine solche Verfahrensregelung könnte der 2001 erarbeitete konkrete Entwurf für ein „Gesetz zur Durchsetzung der Gleichstellung von Frauen und Männern in der Privatwirtschaft" sein. ${ }^{8}$ Seine Umsetzung ist im Jahre 2013 so aktuell und geboten wie schon vor über zehn Jahren. Als Voraussetzung und Grundlage jeder strukturellen Gleichstellungspolitik würde eine solche gesetzliche Regelung die Unternehmen zunächst zu einer jährlich fortzuschreibenden Bestandsaufnahme hinsichtlich der Anteile der Geschlechter in den unterschiedlichen Bereichen verpflichten. ${ }^{9}$ Aufbauend auf der Bestandsaufnahme müssten die Unternehmen geeignete Handlungsfelder auswählen und Gleichstellungsmaßnahmen planen sowie die Umsetzung der Ziele kontrollieren. Um Kompetenz und Kreativität der betrieblichen Akteurinnen und Akteure zu unterstützen, sollten Geschäftsführung, Betriebsrat und Gewerkschaft eine betriebliche Koordinierungsstelle für Gleichstellungsfragen oder eine „, betriebliche Gleichstellungsbeauftragte“ an die Seite gestellt bekommen.

Unternehmen könnten in der Folge auch verpflichtet werden, bei der Beauftragung von Zulieferern und Subunternehmen auf Gleichstellungsprogramme in deren Betrieben zu achten, und bei einer ihnen erkennbaren Verletzung von Diskriminierungsverboten mit in die Haftung genommen werden.

\section{Der djb fordert}

- eine gesetzliche Verpflichtung zu einer geschlechtsdifferenzierten Bestandsaufnahme im Betrieb,

7 BVerfG v. 28.5.1993, BVerfGE 88, 203, 260; siehe auch § 6 der Sozialpartner-Rahmenvereinbarung zur EU-Elternurlaubsrichtlinie 2010/18/EU (die Umsetzungsfrist ist am 8. März 2012 abgelaufen); vgl. Nassibi, Ghazaleh/Wenckebach, Johanna/Zeibig, Nadine, djbZ 2012, S. $111 \mathrm{ff}$.

8 Vgl. Pfarr, Heide M./Weber, Ingrid/Rust, Ursula/Schiek, Dagmar/ Laskowski, Silke/Kocher, Eva, Entwurf der Expertinnenkommission eines 2. Gesetzes zur Durchsetzung der Gleichstellung von Frauen und Männern, in: Pfarr, Heide M. (Hg.), Ein Gesetz zur Gleichstellung der Geschlechter in der Privatwirtschaft, 2001, S. 11 ff. und die Begründung hierzu S. $37 \mathrm{ff}$.

9 S.a. Art. 21 Abs. 4 der Geschlechtergleichbehandlungsrichtlinie 2006/54/EG, wonach die Arbeitgeber ersucht werden sollen, den Arbeitnehmern und/oder den Arbeitnehmervertretern in regelmäßigen angemessenen Abständen Informationen über den Anteil von Frauen und Männern auf den unterschiedlichen Ebenen des Betriebs, ihr Entgelt sowie Unterschiede beim Entgelt zu geben. 
- eine gesetzliche und fristgebundene Verpflichtung, eine betriebliche Koordinierungsstelle für Gleichstellung einzurichten und betrieblich angepasste positive und zielgebundene Gleichstellungsmaßnahmen zu planen und umzusetzen,

- spürbare Sanktionen bei Verstößen sowie eine entsprechende Klagemöglichkeit für Gewerkschaften, Antidiskriminierungsverbände und die Antidiskriminierungsstelle des Bundes.

b) Gleichstellungskriterien bei Vergabe öffentlicher Aufträge Als flankierendes Anreizinstrument für betriebliche Gleichstellungspolitiken bedarf es einer Koppelung öffentlicher Aufträge und Beihilfen des Bundes an die Einhaltung gesetzlicher Gleichstellungspflichten. Der Zugang zu öffentlichen Aufträgen und Beihilfen des Bundes sollte nur für Unternehmen möglich sein, die bei der Abgabe ihres Angebots bzw. bei Antragstellung eine schriftliche Erklärung abgeben, die Benachteiligungsverbote und die gesetzlichen Gleichstellungspflichten einzuhalten. Als Nachweis kann ein Zertifikat über ein erfolgreich durchgeführtes, freiwilliges Gleichstellungsauditverfahren dienen.

\section{Der djb fordert}

- eine Koppelung der Vergabe öffentlicher Aufträge an die Einhaltung gesetzlich vorgeschriebener Regelungen zu Diskriminierungsschutz und Gleichstellungsförderung.

\section{B. Gleichstellung im öffentlichen Dienst}

Der öffentliche Dienst ist in vieler Hinsicht verpflichtet, in Gleichstellungsfragen mit gutem Beispiel voranzugehen. Besonders erinnert sei an die Verpflichtungen, die sich als vertragsschließender Mitgliedstaat der EU ergeben. Wenn der Schwerpunkt dieser Wahlprüfsteine auf der Gleichstellung in der Privatwirtschaft liegt, heißt das nicht, dass im öffentlichen Dienst alles zum Besten stünde. Im Gegenteil - nicht verwirklichte Entgeltgleichheit und bestehende gläserne Decken und Wände bleiben drängende Aufgaben. So muss zum Beispiel ein Gesetz zur Durchsetzung des Entgeltgleichheitsgebotes für Frauen und Männer auch den öffentlichen Dienst einbeziehen. Hier und anderswo sollte der Staat endlich mit tatkräftigem Beispiel vorangehen!

\section{Kommission Zivil-, Familien- und Erbrecht, Recht anderer Lebensgemeinschaften'}

\section{Überprüfung des ehelichen Güterrechts}

Das geltende Recht unterscheidet den Güterstand der Zugewinngemeinschaft (gesetzlicher Güterstand), den Güterstand der Gütertrennung (ein Güterstand ohne güterrechtliche Auswirkungen) und den der Gütergemeinschaft, der - ebenso wie die Gütertrennung - eine vertragliche Vereinbarung der Ehegatten voraussetzt.

Nach einer durch das Bundesministerium für Familie, Senioren, Frauen und Jugend in Auftrag gegebenen Studie wünschen sich Frauen und Männer, dass ihre Ehe ein Leben lang hält, sind sich aber der Tatsache bewusst; dass dies der Realität nicht (immer) entspricht.

Einem großen Anteil der Bevölkerung und der Verheirateten ist dabei - unabhängig von ihrer Ausbildung - die Begrifflichkeit, die mit dem Ehegüterrecht in Zusammenhang steht, unbekannt.

Über den gesetzlichen Güterstand der Zugewinngemeinschaft gibt es sogar schlicht „falsche“ Vorstellungen. So glauben 89 Prozent, dass alles, was während der Ehe erworben wird, beiden Partnern gleichermaßen gehört (93\% der Frauen/87\% der Männer) und 61 Prozent vermuten, dass das gesamte Vermögen „per se“ beiden Partnern gemeinsam gehört (69\% der Frauen/62\% der Männer - sämtlich zitiert aus der Studie: „Partnerschaft und Ehe - Entscheidungen im Lebensverlauf, 2010, S. 11, 49 und 50).

Davon ausgehend gibt es - nach Auffassung des djb - Reformbedarf im ehelichen Güterrecht. Denn die Zugewinngemeinschaft ist ausgerichtet auf die Beendigung der Ehe, nicht auf ihren „Bestand“. Dieser Aspekt sollte indes mit Blick auf die Ergebnisse der vorstehend erwähnten Studie nicht vernachlässigt werden.

Der djb spricht sich daher für den - in seinen Grundzügen gesetzlich geregelten - Güterstand aus, dessen „Leitbild“ nicht (allein) auf die Beendigung der Ehe/Lebenspartnerschaft ausgerichtet ist und gegebenenfalls (ehe-)vertraglich vereinbart werden kann (Wahlgüterstand).

Die Errungenschaftsgemeinschaft kann und sollte Vorbild für Reformüberlegungen sein, zumal dieser Güterstand in zahlreichen europäischen Mitgliedstaaten, darunter unter anderem Italien, Spanien und Frankreich, durch die Eheschließung begründet wird.

\section{Nachehezeitlicher Unterhalt}

Die zum 1. März 2013 in Kraft getretene Reform des Unterhaltsrechts ist mitnichten eine Überarbeitung des geltenden Rechts, sondern eine Klarstellung im Rahmen einer Einzelnorm, die der Rechtsprechung des Bundesgerichtshofs folgt. Der djb hat darauf unter anderem in einer Presseerklärung aufmerksam gemacht (http://www.djb.de/Kom/K2/pm13-5/).

Der Unterhaltsanspruch der oder des Unterhaltsberechtigten (in der Regel noch immer die Frau) hängt - wie bisher maßgeblich von Billigkeitserwägungen und damit von einer Einzelfallprüfung ab. Zurückzuführen ist dies auf die grundsätzlichen Änderungen des Unterhaltsrechts zum 1. Januar 2008, die in ihrer Konsequenz eine Vielzahl unbestimmter und damit auslegungsbedürftiger Rechtsbegriffe gebracht haben.

Die/der Unterhaltsberechtigte ist nunmehr gehalten umfassend vorzutragen. Denn für den Unterhaltsanspruch entscheidend ist, was zu den eigenen Lebensverhältnissen und dem eigenen Werdegang (prognostisch) vorgetragen und bewiesen werden kann. Die Verfahren sind im nachehelichen Unterhalt

1 Redaktion: Brigitte Meyer-Wehage, Komm. Vorsitzende der Kommission Zivil-, Familien- und Erbrecht, Recht anderer Lebensgemeinschaften. 
„von langer Dauer“ angesichts der das Unterhaltsrecht beherrschenden Regel-Ausnahme-Verhältnisse und der daraus folgenden Darlegungs- und Beweislast; gelegentlich noch zugespitzt auf die sogenannte sekundäre Darlegungslast.

Die Entscheidungen der Gerichte sind angesichts des „Einzelfalls“ eher von „Beliebigkeit“ als von Rechtssicherheit geprägt (von der Personalbindung einmal abgesehen).

\section{Der djb fordert daher}

- eine ergebnisoffene Diskussion zur „Reform der Reform“ unter Beachtung vorangestellter Erwägungen, die den Änderungsbedarf konkretisieren und in Vorschlägen zusammenführen.

\section{Unterhaltsvorschuss}

Das Gesetz zur Änderung des Unterhaltsvorschussgesetzes und anderer Gesetze (Unterhaltsvorschussentbürokratisierungsgesetz) hat im Ergebnis, sofern es denn in Kraft treten sollte, seinen Zweck verfehlt.

Die Kernpunkte des Gesetzes, die nach überwiegender Auffassung reformbedürftig sind, nämlich die Festschreibung, dass Leistungen nur bezogen werden können, soweit das Kind das 12. Lebensjahr nicht vollendet hat, und die Bezugsdauer von maximal 72 Monaten, sind unverändert geblieben.

Damit sind wesentliche Überlegungen, die nicht zuletzt der Stärkung Alleinerziehender (überwiegend Frauen) dienen, zurückgestellt worden. Alleinerziehende sind jedoch häufiger von Armut bedroht und damit auf Förderung angewiesen. Die Betreuungssituation von Kindern, die älter als 12 Jahre sind, ist sogar häufig schwierig als die jüngerer Kinder, da Ganztagsschulen flächendeckend nicht ausreichend zur Verfügung stehen.

\section{Vertretung durch den anderen Elternteil bei Kindesunterhalt} Die Vertretungsregelung des $\mathbb{S} 1629$ Absatz 2 Satz 2 BGB bei gemeinsamer elterlicher Sorge - ist zu überarbeiten. Denn in der Praxis beruft sich der unterhaltspflichtige Elternteil, der sich in die Betreuung des gemeinsamen Kindes einbringt (regelmäßig der Kindesvater), häufig auf eine fehlende Vertretungsbefugnis des anderen Elternteils (regelmäßig die Kindesmutter) zur Geltendmachung von Barunterhaltsansprüchen (Stichwort: Wechselmodell).

Bevor ein/e Ergänzungspfleger/in bestellt werden kann, ist aber zunächst zu prüfen, ob die Voraussetzungen vorliegen, d.h. die Klärung der Betreuungsanteile „blockiert“ das (eigentliche) Unterhaltsverfahren mit allen daraus resultierenden Folgen.

- Der djb fordert insoweit Abhilfe.

\section{Adoptionsrecht}

Nach der Entscheidung des Bundesverfassungsgerichts in den Verfahren $1 \mathrm{BvL}$ 1/11 und $1 \mathrm{BvR}$ 3247/09 ist das Adoptionsrecht im Recht anderer Lebensgemeinschaften zu ändern.

Das geltende Recht führt zu einer Ungleichbehandlung (auch) der betroffenen Kinder im Vergleich zu denjenigen, die in einer Ehe leben.

\section{Der djb fordert}

- - entsprechend den zeitlichen Vorgaben des Bundesverfassungsgerichts - eine zügige Umsetzung des Gesetzesvorhabens, um im Bereich der Adoption die notwendige Rechtssicherheit zu schaffen.

\section{Vertrauliche Geburt}

Der Referentenentwurf aus dem Bundesministerium für Familie, Senioren, Frauen und Jugend befindet sich im Gesetzgebungsverfahren. Ob er in der laufenden Legislaturperiode noch verabschiedet werden wird, ist offen.

Der djb hat in seiner Stellungnahme vom 26. November 2012 (http://www.djb.de/Kom/K2/st12-11/) Bedenken gegen den Entwurf vorgebracht, insbesondere zum Widerspruchsrecht der Mutter, hält jedoch eine gesetzliche Regelung für notwendig, um anonyme Geburten und Babyklappen alsbald überflüssig zu machen.

\section{Der djb fordert daher}

- eine zeitnahe gesetzliche Regelung der vertraulichen Geburt.

\section{Erbrecht}

\2057a BGB regelt die Ausgleichspflicht besonderer Leistungen eines Abkömmlings für die/den Erblasser/in.

Die beschriebenen Leistungen, insbesondere im Bereich der Pflege, erbringen jedoch häufig die Schwiegertöchter.

Der djb sieht im fehlenden Ausgleich eine Benachteiligung und fordert

- eine entsprechende Regelung zugunsten der pflegenden Ehefrauen/Lebenspartnerinnen.

\section{Kommission Strafrecht und Fachkommission Gewalt gegen Frauen und Kinder}

Als einer der großen Mitgliedstaaten der Europäischen Union ist Deutschland gehalten, den mit allen gemeinsam vereinbarten Raum der Freiheit, der Sicherheit und des Rechts in Europa nicht nur mit zu gestalten, sondern auch selbst vorbildhaft umzusetzen. Dazu gehört im Strafrecht der Schutz der Bürgerinnen und Bürger vor Straftaten (Prävention) und im Falle der Opferwerdung die Gewährleistung von Zugang zum Recht, Schutz, Hilfe und Ausgleich. Dies gilt insbesondere für Frauen und Mädchen, die bei Straftaten gegen Leib, Leben, Freiheit, Ehre und sexuelle Selbstbestimmung in noch immer deutlich höherer Zahl Opfer von Straftaten werden als Männer.

Politisch heißt dies, Prävention in die Rechtspolitik einzubinden, bereits erlassene Richtlinien der EU zeitgerecht um-

1 Redaktion: Dagmar Freudenberg, Vorsitzende der Kommission Strafrecht. 
zusetzen, Vereinbarungen wie die Europarats-Konvention gegen Gewalt gegen Frauen und häusliche Gewalt nicht nur zu unterzeichnen, sondern auch zu ratifizieren, sowie deutsches Recht bereits ratifizierten Vereinbarungen wie der UNKinderrechts-Konvention vollständig anzupassen.

\section{Menschenhandel}

Nach der aktuellen Studie der EU von 2013 zum Ausmaß des Menschenhandels in den Jahren 2008 bis 2010 hat die Zahl der Opfer von Menschenhandel um 18 Prozent zugenommen, etwa 70 Prozent der Opfer sind Frauen und Mädchen. Die zur Umsetzung der Richtlinie 2011/36/EU des Europäischen Parlaments und des Rates vom 5. April 2011 zur Verhütung und Bekämpfung des Menschenhandels und zum Schutz seiner Opfer in Deutschland im Strafrecht und Strafprozessrecht bisher vorgenommen Änderungen sind unvollkommen. ${ }^{2}$ Instrumente der Zeugenschutzprogramme sind für die Opfer häufig keine sinnvollen Schutzangebote. Zudem ist eine verstärkte, phänomenbezogene Fortbildung der diese Fälle bearbeitenden Professionellen in allen Bereichen noch immer nicht gewährleistet.

\section{Der djb fordert,}

- unterhalb der Schwelle des Zeugenschutzgesetzes zeugenschutzähnliche Maßnahmen zu erarbeiten und den betroffenen Opfern anzubieten sowie

- Aus- und Fortbildung aller diese Fälle bearbeitenden Professionen, insbesondere in Polizei und Justiz, dem phänomenbezogenen Bedarf entsprechend flächendeckend zu gewährleisten.

\section{Sexualdelikte}

Das deutsche Sexualstrafrecht, zuletzt grundlegend reformiert im Jahr 1997, orientiert sich grundsätzlich einerseits am Schutz bestimmter Opfergruppen wie Kinder (bis 18 Jahre), Widerstandsunfähige und Abhängige in bestimmten Überund Unterordnungsverhältnissen, und andererseits allgemein an bestimmten konstellativen Faktoren bei der Durchführung sexueller Handlungen, wie Gewalt, Drohung mit Gefahr für Leib oder Leben oder einer schutzlosen Lage. Gemäß Artikel 36 des Übereinkommens des Europarats zur Verhütung und Bekämpfung von Gewalt gegen Frauen und häuslicher Gewalt, das von Deutschland zwar unterzeichnet, aber noch nicht ratifiziert wurde, wird von den Europarats-Mitgliedern erwartet, Maßnahmen dahingehend zu ergreifen, dass nicht einverständliche sexuelle Handlungen unter Strafe stehen. In England und Wales sowie Norwegen gibt es bereits vergleichbare Regelungen. ${ }^{3}$

\section{Der djb fordert,}

- das Übereinkommen des Europarates zur Verhütung und Bekämpfung von Gewalt gegen Frauen und häuslicher Gewalt $\mathrm{zu}$ ratifizieren und

- die Sexualdelikte dahingehend zu reformieren, dass nicht einverständliche sexuelle Handlungen grundsätzlich unter Strafe gestellt werden.

\section{Verbesserung der Nutzung der Videovernehmung im Strafverfahren zum Schutz der (mutmaßlichen) Opfer vor sekundärer Viktimisierung}

Mit der im März 2013 erfolgten Beschlussfassung über das Gesetz zur Stärkung der Rechte von Opfern sexuellen Missbrauchs (StORMG) hat der Bundestag einige der Forderungen des Runden Tisches „Sexueller Kindesmissbrauch in Abhängigkeits- und Machtverhältnissen in privaten und öffentlichen Einrichtungen und im familiären Bereich “ aufgegriffen. Die vorgenommenen Änderungen reichen jedoch nicht aus:

Zur Vermeidung von Mehrfachvernehmungen sind die Vorschriften zur Videovernehmung in den Paragrafen 58a und 255a StPO ergänzt worden. Eine Videovernehmung soll danach auch erfolgen, wenn „damit die schutzwürdigen Interessen von Personen unter 18 Jahren sowie von Personen, die als Kinder oder Jugendliche durch eine der in $\$ 255$ a Absatz 2 genannten Straftaten verletzt worden sind, besser gewahrt werden können.“

Diese Änderung war überfällig, um auch für diejenigen Verletzten die Videovernehmung ausdrücklich vorzusehen, die bei der Anzeigenerstattung als Erwachsene den in ihrer Kindheit erlebten sexuellen Missbrauch berichten. Diese Verletzten sind häufig bis in das späte Erwachsenenalter traumatisiert und nicht in der Lage, die Geschehnisse mehrfach detailliert zu berichten. Die Aufnahme per Bild-Ton-Träger (Videovernehmung) ist geeignet, in diesen Fällen die Anzahl der Vernehmungen zu reduzieren. Die noch immer vielerorts fehlende Nutzung des Instruments der Videovernehmung gründet sich jedoch zumeist in der mangelnden Akzeptanz aufgrund der fehlenden Praktikabilität der Umsetzung der Videovernehmung nach dem geltenden Recht. So fehlt es zum einen noch immer an ausreichender Schulung und Fortbildung der vorgesehenen Vernehmungspersonen. Zum anderen ist die Problematik nicht gelöst, dass die derzeitigen Protokollvorschriften moderner Technik nicht angepasst sind. Die derzeit bestehende Verpflichtung, eine vollständige Abschrift der Videovernehmung zu den Akten zu nehmen, bindet erhebliche personelle Ressourcen.

\section{Der djb fordert deshalb}

- eine Reform der Protokollvorschriften, zum Beispiel in der Weise einer Änderung des $\mathbb{S} 168$ a Absatz 2 StPO dahingehend, dass im Fall der Zeugen- oder Beschuldigtenvernehmung mit Aufzeichnung mittels Tonaufnahmegerät oder mittels Bild-Ton-Träger eine wortgetreue Aufnahme der wesentlichen, den Sachverhalt betreffenden Passagen in das Protokoll ausreichend ist, sofern eine Speicherung auf Datenträger erfolgt. ${ }^{4}$

2 Vgl. auch die Wahlprüfsteine der Kommission Öffentliches Recht, Europa- und Völkerrecht des djb in diesem Heft, S. 66.

3 Vgl. den Aufsatz von Sabine Kräuter-Stockton, $\$ 177$ StGB (Vergewaltigung/Sexuelle Nötigung) - Kritik und Verbesserungsvorschläge im Vergleich mit den Regelungen in Norwegen, Schweden und England/Wales, in diesem Heft, S. 89.

4 Vgl. die Stellungnahme St 11-1 des djb v. 14.2.2011 unter <http://www.djb.de/Kom/K3/St11-1/> (Zugriff: 17.4.2013). 


\section{Schutz von älteren Menschen vor Gewalt und Vermö- gensdelikten}

Die demografische Entwicklung in Deutschland belegt, dass immer mehr Ältere in unserer Gesellschaft leben, darunter wegen der höheren Lebenserwartung in überwiegender Anzahl Frauen. Straftaten, durch die ältere Menschen geschädigt werden, nehmen aufgrund der demografischen Entwicklung weiterhin an Bedeutung zu. Es handelt sich hier, insbesondere wenn es um hochaltrige Geschädigte geht, um ein Deliktsfeld, welches in der Öffentlichkeit kaum in Erscheinung tritt. Die Geschädigten sind als Zeugen oft „unsichtbar“ - sie schämen sich dafür, Opfer geworden zu sein und erstatten nur selten Anzeige, weil sie damit eine Schwäche, die ausgenutzt wurde, eingestehen müssten. Sie wollen aber auch oft nahestehende Personen, von denen sie im Rahmen der Pflege abhängig sind, oder gar Angehörige nicht als Täter/in benennen. Unter Umständen sind sie auch körperlich und intellektuell nicht dazu in der Lage, die Tat zu ihrem Nachteil zu schildern. Diese günstigen Tatbegehungs- und Tatverdeckungsmöglichkeiten nutzen Täter/innen gezielt aus - sowohl wenn es um Gewalt geht, die überwiegend von nahestehenden Personen begangen wird, als auch bei Vermögensstraftaten, bei denen ältere Menschen sowohl durch Angehörige (z.B. Untreue) geschädigt werden, als auch von völlig Fremden im Rahmen von Betrugshandlungen gezielt ausgewählt werden (z.B. sog. „Enkeltrick)“. In diesem Zusammenhang gilt es, Schutzmöglichkeiten zu kreieren und die als Hilfs-, Ermittlungs- und Kontrollinstanzen tätigen Professionellen phänomengerecht aus- und fortzubilden.

\section{Der djb fordert}

- die Prüfung der Einführung eines Straftatbestandes der Ausnutzung der altersbedingten Abwehrunfähigkeit gegen Vermögensschädigung sowie

- die phänomengerechte Aus- und Fortbildung aller in Hilfs-, Ermittlungs- und Kontrollinstanzen tätigen Professionellen.

\section{Mindeststandards für Opfer von Straftaten}

Am 25. Oktober 2012 wurde die Richtlinie der EU zu Mindeststandards für Opfer von Straftaten 2012/29/EU verabschiedet. Die Umsetzung muss - auch in Deutschland - bis zum 15. November 2015 erfolgen. Einige der dort verankerten Rechte sind in Deutschland bereits geltendes Recht. Dies gilt jedoch nicht für alle Maßnahmen. So ist ein Anspruch auf qualifizierte Dolmetschleistung oder Übersetzungsleistung insbesondere für weibliche Opfer von Straftaten im Bereich der Gewalt- und Sexualstraftaten noch nicht verankert. Zwar gibt es bereits einen Gesetzentwurf zur Umsetzung der EU-Richtlinie 2010/64/EU zu Dolmetscher- und Übersetzerleistungen für Beschuldigte. Indessen fehlen dort allgemein entsprechende Regelungen zur Sicherung der Qualität und insbesondere Anspruchsgrundlagen für die Opfer von Straftaten, die der deutschen Sprache nicht hinreichend mächtig sind.

Darüber hinaus werden durch die aktuellen Diskussionen insbesondere der Bundesländer zur Einschränkung der Ver- fahrenskostenhilfe aus Haushaltsgründen die in der Richtlinie verankerten Rechte der Opfer von Straftaten auf kostenfreien Zugang zum Recht sowie zu Unterstützung, Begleitung und Schutz vor erneuter Viktimisierung, Einschüchterung und Vergeltung erheblich gefährdet.

\section{Der djb fordert,}

- die EU-Opferschutzrichtlinie zeitgerecht umzusetzen und insbesondere das Recht auf qualifizierte und umfassende Dolmetscher- und Übersetzungsleistungen für Opfer von Straftaten sicherzustellen sowie

- die Verfahrenskostenhilfe nicht zu Lasten der Opfer von Straftaten einzuschränken.

\section{Schutz vor häuslicher Gewalt}

Um häusliche Gewalt in der Gesellschaft zu bekämpfen und Opfer von häuslicher Gewalt, in der Mehrzahl Frauen und die in den Beziehungen lebenden Kinder, zu schützen und zu begleiten hat Deutschland bereits viele notwendige Schritte unternommen. Anhand seiner Länderumfrage 2011 zum zehnjährigen Bestehen des Gewaltschutzgesetzes hat der djb gleichwohl noch viele Mängel festgestellt, die es zu beseitigen gilt. Eine der wichtigsten Reformen ist die Anhebung des Strafrahmens in $\mathbb{S} 4$ GewSchG. Der derzeitige Strafrahmen von Geldstrafe oder Freiheitsstrafe bis zu einem Jahr entspricht dem Strafrahmen der Fischwilderei und vermittelt in der Gesellschaft den Eindruck, es handele sich um ein Bagatelldelikt. Sogar die Nachstellung („Stalking“) in $\$ 238$ StGB hat einen höheren Strafrahmen von Geldstrafe oder Freiheitsstrafe bis zu drei Jahren.

Darüber hinaus muss, um der Europarats-Konvention von 2011 zu Gewalt gegen Frauen und häuslicher Gewalt ebenso wie der EU-Richtlinie Mindeststandards 2012/29/EU Genüge zu tun, das Durcheinander von Definitionen in diesem Bereich beendet werden. Ab 2017 muss Deutschland wie jeder andere Mitgliedstaat der EU vergleichbare Daten an die EU mitteilen. Dies bedingt eine einheitliche Definition im Bereich der häuslichen Gewalt, eine Neubewertung des bei der Bekämpfung dieses gesellschaftlichen Phänomens erforderlichen Arbeitsaufwands in allen Professionen und eine dementsprechende Bereitstellung insbesondere personeller Ressourcen.

Schließlich gilt es, den in der EU-Richtlinie Mindeststandards 2012/29/EU vorgesehenen Anspruch der Opfer von Straftaten auf kostenfreien Zugang zu Schutz vor erneuter Viktimisierung, Einschüchterung und Vergeltung dadurch zu gewährleisten, dass die Finanzierung von Opferschutzeinrichtungen bundesweit sichergestellt wird.

\section{Der djb fordert,}

- den derzeit geltenden Strafrahmen des $\mathbb{} 4$ GewSchG unrechtsangemessen anzuheben,

- im Bereich häusliche Gewalt für alle beteiligten Professionen einheitliche und vergleichbare Definitionen für Deutschland zu schaffen, die mit den Definition in anderen EU-Staaten kompatibel sind, 
- eine dem Aufwand bei der Bekämpfung des gesellschaftlichen Phänomens häusliche Gewalt angemessene Bereitstellung personeller Ressourcen in allen betroffenen Professionen und
- die bundesweite Sicherstellung der Finanzierung von Opferschutzeinrichtungen.

\section{Kommission Recht der sozialen Sicherung, Familienlastenausgleich'}

\section{Einkommensbesteuerung und Kindergeld}

Der Berücksichtigung von Ehe und eingetragener Lebenspartnerschaft sowie der Familie im Einkommensteuerrecht ist nach wie vor nicht konsistent. Es fehlt an einem einheitlichen Konzept, das den Belangen von Eltern mit Kindern Rechnung trüge, ohne sie zu bloßen Funktionsträgern zu degradieren. Das Ehegattensplitting begründet deutliche Fehlanreize zulasten der Erwerbstätigkeit von Frauen. Dies ist auch nicht nur eine Frage des volkswirtschaftlich und arbeitsmarktpolitisch begründeten Bedarfs an weiblichen Beschäftigten. Vielmehr bestehen Widersprüche zum geltenden Unterhaltsrecht, das nach der Trennung und Scheidung einer Ehe viel stärker auf die eigenständige Existenzsicherung der Eheleute setzt als vor 2008. Auch das Grundsicherungsrecht gründet auf dem Leitbild des „adult worker“ und nimmt hiervon erziehende und betreuende Eltern nur in den ersten drei Lebensjahren eines Kindes aus. Es kann und darf daher im Einkommensteuerrecht nicht so getan werden, als begründete die Ehe immer noch eine langjährige Sicherung der materiellen Existenz für einen Ehepartner, der zugunsten der Ehe und/oder der Betreuung von Kindern auf eine existenzsichernde Erwerbstätigkeit verzichtet.

Bei Betrachtung des rechtlichen Gesamtsystems wird die Beibehaltung der Lohnsteuerklasse V und die unterbleibende Berücksichtigung von geringfügiger Beschäftigung („Minijobs“) bei der Einkommensteuer eines Ehepaares vollends unverständlich. Geringfügige Beschäftigung bis zu einem $\mathrm{Zu}$ verdienst von 450 Euro bleibt damit steuerlich günstig, während jeder weitere verdiente Euro mit den vollen Abzügen der Eheleute belegt werden kann. Die hohe Besteuerung des geringeren Verdienstes verringert dessen Wert gegenüber dem in Steuerklasse III besteuerten höheren Einkommen zusätzlich. Die künstliche Hürde des (Wieder-)Einstiegs in eine existenzsichernde Beschäftigung wird „erkauft“ mit einem steuerlichen Darlehen an die Eheleute, die dieses erst mit der Einkommensteuerveranlagung als Steuernachzahlung „zurückzahlen“ müssen. Der Staat subventioniert hiermit eine der Gleichstellung zuwiderlaufende Form der Einkommensbesteuerung.

Zugleich enthält dasselbe System den Eltern von betreuungsbedürftigen Kindern nach wie vor die Abzugsfähigkeit von erwerbsbedingten Betreuungskosten in einem angemessenen, die erforderlichen Ausgaben realitätsgerecht berücksichtigenden Umfang vor.

\section{Der djb fordert vor diesem Hintergrund}

- die Ablösung des Ehegattensplittings durch eine Individualbesteuerung mit übertragbarem Grundfreibetrag und gleichzeitig eine angemessene Berücksichtigung des Tatbestands „Kind/er“ im Einkommensteuer- bzw. Kindergeldrecht,
- in einem ersten Schritt jedoch zumindest die Abschaffung der Steuerklasse V im Lohnsteuerverfahren sowie

- die Absetzbarkeit sämtlicher erwerbs- und ausbildungsbedingter Kinderbetreuungskosten vom ersten Cent an als Werbungskosten bzw. Betriebsausgaben. Nicht erwerbsbedingte Kinderbetreuungskosten können als Sonderausgaben Berücksichtigung finden.

Nach wie vor berücksichtigt der Steuergesetzgeber zu wenig, dass von der hohen Umsatzsteuer Familien besonders schwer betroffen werden. Sie haben viele und spezifische Ausgaben, die regelmäßig mit dem vollen Umsatzsteuersatz belegt sind. Die steuerlichen Entlastungen bei der Einkommensteuer durch die Freibeträge nach $\mathbb{S} 32 \mathrm{EStG}$, aber auch die Vergünstigungen des Ehegattensplittings, finanzieren folglich viele Familien durch die Umsatzsteuer mit.

\section{Der djb fordert}

- die Prüfung der Verwirklichung von niedrigeren Mehrwertsteuersätzen für Baby- und Kleinkindprodukte im europäischen Raum.

\section{Krankenversicherung}

Die beitragsfreie Mitversicherung in der gesetzlichen Krankenversicherung (GKV) verstärkt die negativen Effekte des Ehegattensplittings und der geringfügigen Beschäftigung. „Minijobs“ von Ehefrauen als Zuverdienst-Beschäftigung werden mit ihr aus der gesetzlichen Krankenversicherung heraus subventioniert. Angesichts der problematischen Effekte der geringfügigen Beschäftigung auf Einkommens- und Karrierechancen sowie auf die materielle Sicherung der Frauen im Alter ist eine solche Subvention nicht zu rechtfertigen. Schutzbedürftig sind außerdem nicht die Eheleute, sondern Eltern mit minderjährigen Kindern. Daher sollte eine grundlegende Neuorientierung der Beitragsfreiheit an dem Tatbestand des Aufziehens von Kindern erfolgen (ähnlich, wie dies bereits für die Pflege geschieht). Um einen sinnvollen Anschluss zu gewährleisten, sollte eine freiwillige Versicherung in der GKV nach dieser Zeit ermöglicht werden für den Fall, dass der betreuende Elternteil weiterhin keiner Erwerbstätigkeit nachgeht.

\section{Der djb fordert}

- die beitragsfreie Mitversicherung von Eheleuten nach $\mathbb{} 10$ SGB V durch eine zeitlich befristete beitragsfreie Versiche-

\footnotetext{
1 Redaktion: Prof. Dr. Margarete Schuler-Harms, Vorsitzende der Kommission Recht der sozialen Sicherung, Familienlastenausgleich des djb.
} 
rung von Eltern in der gesetzlichen Krankenversicherung abzulösen und eine anschließende Versicherungsmöglichkeit in der GKV auf freiwilliger Basis vorzusehen. Ein Zeitraum der Beitragsfreiheit von drei Jahren wäre im Vergleich mit dem Bundeselterngeld- und -elternzeitgesetz, mit dem zivilrechtlichen Unterhaltsrecht und dem Recht der Grundsicherung sachgerecht.

In der privaten Krankenversicherung (PKV) werden aufgrund europäischer Vorgaben nunmehr für Frauen und Männer gleiche Tarife angeboten. Allerdings werden Bestandsverträge nicht umgestellt. Der Wechsel in einen neuen Unisex-Tarif wird von den meisten Versicherern durch eine neue Leistungsgestaltung verstellt. Das bedeutet für alle bisher privat Krankenversicherten, dass sie in den geschlechterdifferenzierten Tarifen gefangen bleiben und Unisex in der privaten Krankenversicherung nicht erst nach Jahrzehnten verwirklicht wird.

\section{Der djb fordert,}

- die Versicherungswirtschaft zur Umstellung von Bestandsverträgen gesetzlich zu verpflichten.

\section{Der djb fordert in diesem Zusammenhang außerdem}

die Öffnung der GKV für Beamtinnen und Beamte.

Anstelle einer Umstellung der PKV-Bestandsverträge auf Unisex kann die Gleichstellung der Geschlechter auch dadurch verwirklicht werden, dass die GKV für diejenigen geöffnet wird, die keinen Unisex-Vertrag bei der PKV erhalten. Dies ist für Beamtinnen und Beamte allerdings nur dann sinnvoll, wenn in der GKV für diese Wechsler beihilfefähige Tarife offenstehen, wie sie heute für sogenannte DO-Angestellte existieren. Die kalkulierten Alterungsrückstellungen der wechselnden Versicherten sind dabei zum Ausgleich der Risikoselektion auf den Gesundheitsfonds zu übertragen.

Gerade für teilzeitbeschäftigte Beamtinnen und Beamte unterer Lohngruppen ist sowohl die einkommensunabhängige PKV als auch die GKV-Versicherung zum Höchstsatz ohne Arbeitgeberanteil sozial ungerecht.

\section{Alterssicherung}

Art und Maß der Sicherung materieller Lebensgrundlagen im Alter resultiert regelmäßig aus der Lebens- und Erwerbsbiografie. Die steuer- und sozialrechtliche Begleitung der Verbindung von Erwerbsarbeit und Familienleben ist deshalb auch für die Alterssicherung von großer Bedeutung.

Daher setzt vor allem die rentenrechtliche Privilegierung geringfügiger Beschäftigung falsche Anreize, auf eine eigenständige Altersvorsorge zu verzichten. Sie ist daher zu beseitigen.

Grundsätzlich begrüßenswert ist die Verzahnung von Grundsicherung im Alter und Rentenansprüchen, wie sie in der gegenwärtigen Legislaturperiode versucht wurde. Allerdings waren die Voraussetzungen nicht durchdacht. Der djb begrüßt es, wenn rentenrechtlich Lebensbiografien von Frauen so aufgewertet werden, dass die Rentenleistung über der Grundsicherung liegt. Die Berücksichtigung privater Altersvorsorge ist hierbei jedoch systemwidrig und dysfunktional, da sie gerade wegen diskontinuierlicher Erwerbsbiografien typischerweise noch lückenhafter ist als die Rentenbiografie.

\section{Der djb fordert,}

- die Kindererziehungszeiten für vor 1992 geborene Kinder zu erhöhen.

Die Hinterbliebenenrente ist weiter zu reformieren. Die Differenzierung zwischen Eheleuten und eingetragenen Lebenspartnern einerseits und nichtehelicher Elternschaft mit Kindern andererseits ist nicht mehr sachgerecht. Auch die Erziehungsrente gehört auf den Prüfstand, da sie geschiedenen Eltern unter Umständen langjährig zugutekommt, anderen Alleinerziehenden aber vorenthalten wird.

\section{Der djb fordert daher,}

- die Hinterbliebenenrente sowie die Erziehungsrente nicht mehr an der Ehe, sondern an der gemeinsamen Elternschaft auszurichten.

\section{Elterngeld}

Veränderungen der geschlechtsspezifischen Rollenverteilung bei der Familienarbeit durch das Elterngeld sind zwar vorangekommen. Der Weg zur gleichen Verteilung der Familienarbeit zwischen Müttern und Vätern ist aber noch weit. Mit weiteren Reformen des Elterngeldes sollte die Politik dazu beitragen, dass Mütter und Väter in der Phase nach der Geburt eines Kindes zusätzliche Handlungsspielräume gewinnen. Verbesserungswürdig ist der gleichzeitige Teilelterngeldbezug beider Eltern, da mit ihm die Flexibilität erhöht wird, sich Beruf und Betreuung zu teilen. Eine Ausweitung der Partnermonate würde Väter in ihrer Rolle als Betreuende ansprechen und längere Erwerbsunterbrechungen auch von Männern selbstverständlicher machen.

\section{Der djb fordert daher,}

- das Elterngeld so weiterzuentwickeln, dass der gleichzeitige Teilelterngeldbezug nicht mehr finanziell benachteiligt wird, - die Partnermonate auszuweiten.

\section{Betreuungsgeld}

Die Einführung des Betreuungsgeldes zum 1. August 2013 ist ein gleichstellungs- und familienpolitischer Rückschritt. Das Betreuungsgeld belohnt junge Eltern finanziell für die Nichtinanspruchnahme öffentlicher Kinderbetreuungsangebote. Die Wahl zwischen Geldleistung und Betreuungsplatz ist aus Sicht des djb keine sinnvolle Anspruchsvoraussetzung in der Familienförderung. Das Betreuungsgeld geht an den realen Bedürfnissen von Familien vorbei. Es begegnet in der konkreten Ausgestaltung darüber hinaus auch erheblichen verfassungsrechtlichen Bedenken. Allen Eltern gebührt Anerkennung für ihre Leistung - unabhängig davon, wie sie die Betreuung ihrer Kinder organisieren. Die Wahlmöglichkeiten von Eltern werden nicht durch ein Betreuungsgeld, sondern durch die Bereitstellung flächendeckender und qualitativ hochwertiger Kinderbetreuungsangebote erhöht. Das Betreuungsgeld sendet hingegen ein familien- wie gleichstellungspolitisch falsches Si- 
gnal. Der Verzicht auf einen Betreuungsplatz kann gerade für Mütter den Wiedereinstieg in den Beruf erschweren und damit der Vereinbarkeit von Familie und Beruf entgegenwirken.

\section{Der djb fordert,}

n das Betreuungsgeld nicht einzuführen bzw. abzuschaffen.

\section{Grundsicherung nach SGB II}

Viele Frauen sind nach dem SGB II sogenannte Nichtleistungsberechtigte. Als solche verlieren sie ihren eigenen Anspruch auf Eingliederungsleistungen nach dem SGB II, wenn die Bedarfsgemeinschaft aus dem ALG-II-Bezug ausscheidet, weil der Partner über ein Einkommen verfügt, das den Bedarf der gesamten Lebens- und Hausgemeinschaft deckt. Nach der Umstellung von der Einsatzgemeinschaft nach BSHG auf die Bedarfsgemeinschaft nach SGB II kann diese Wirkung auch durch einen im Haushalt lebenden Verwandten ausgelöst werden. Wären diese Frauen alleinlebend, so wären sie leistungsberechtigt und umfassend anspruchsberechtigt nach SGB II.

\section{Der djb fordert,}

- die Berechnungsmethode der Bedarfe dahin zu verändern, dass nicht mehr die Bedarfsgemeinschaft, sondern wie nach altem Recht die Einsatzgemeinschaft berücksichtigt wird und zukünftig bei der Bedarfsberechnung der Regelbedarfe die Vertikal- statt der gegenwärtigen Horizontalberechnung Anwendung findet.

Vor allem aber stehen die Einstandspflichten für Partnerkinder (sog. Stiefkinderregelung) im Widerspruch zu zivilrechtlichen Unterhaltspflichten. Sie beeinträchtigen vor allem Alleinerziehende - und damit ganz überwiegend Frauen mit Kindern in ihrer Lebensführung. Einem für sich allein leistungsfähigen Partner wird auch bei nichtehelichem Zusammenleben mit der Mutter die Bedürftigkeit ihrer Kinder zugerechnet mit allen Folgen, die die Einstufung als Bedarfsgemeinschaft nach SGB II mit sich bringt. Die sog. Stiefkinderregelung bewirkt in Verbindung mit dem Institut der Bedarfsgemeinschaft, dass die Eingehung einer Partnerschaft unter Umständen von der Frau oder dem Partner vermieden wird. Gehen die Partner dennoch eine Lebensgemeinschaft ein und kommt es zur Anrechnung des "Stiefkindes“, so hat das Kind in einer nichtehelichen Partnerschaft keinen eigenständigen Anspruch auf Existenzsicherung gegen den Partner der Mutter und profitiert auch nicht von einem Anspruch der Mutter.

\section{Der djb fordert deshalb,}

- die Regelungen im SGB II zu der Einstandspflicht für die Kinder eines nichtehelichen Lebenspartners aufzuheben.

\section{Pflege}

Private Sorgearbeit durch eine politische und gesetzgeberische Gesamtstrategie für informell und semiprofessionell Pflegende besser unterstützen:

Die Pflege von älteren Menschen ist eine gesamtgesellschaftliche Verantwortung. Die Mehrheit der informell, d.h. nicht erwerbsmäßig Pflegenden, die diese oft auch belastende private Sorgearbeit leisten, sind Frauen.

Der djb hält eine politische und gesetzgeberische Gesamtstrategie für informell Pflegende, zum Beispiel im Rahmen eines „Nationalen Aktionsplans“, für erforderlich, um informell Pflegende stärker zu einer eigenen Zielgruppe der Politik und des Sozialrechts zu machen. Die verstreuten unzureichenden Ansprüche und Maßnahmen für informell Pflegende sind zu einem kohärenten System zusammenzuführen.

\section{Der djb fordert,}

- die fachliche und psychosoziale Unterstützung von informell, aber auch semiprofessionell Pflegenden zu verbessern, insbesondere durch verbesserte Leistungsansprüche auf individuell ausgerichtete Anleitung, Schulung und Supervision. Zudem sind ausreichende Angebote der Kurzzeit-, Verhinderungs- und Tages- und Nachtpflege zu schaffen und dauerhaft $\mathrm{zu}$ finanzieren.

Bessere Vereinbarkeit von Pflege und Beruf: Die bisherigen Maßnahmen (PflegeZG und FamilienpflegeZG) haben kaum Wirkung entfaltet. Im Kern geht es jedoch für informell Pflegende, die im Erwerbsleben stehen, um drei Kernthemen:

1. Möglichkeit zur flexiblen Reduktion der Arbeitszeit mit Rückkehranspruch,

2. Existenzsichernde Beschäftigung trotz Pflege (auch mit Blick auf die Alterssicherung) und

3. Vorhandensein einer betrieblichen und gesellschaftlichen Unterstützungsinfrastruktur für die Pflege. Auch die berufliche und gesellschaftliche Teilhabe von informell Pflegenden ist häufig eingeschränkt.

\section{Der djb regt an,}

- die Regelungen des PflegeZG und FamilienpflegeZG unter Berücksichtigung der drei genannten Punkte in das reguläre Arbeits- und Sozialrecht (z.B. TzBfG, ArbZG usw.) zu überführen. Die berufliche und gesellschaftliche Teilhabe von informell Pflegenden ist durch eine solidarische Absicherung sicherzustellen.

Professionelle Pflege - Vollzeitarbeitsplätze ermöglichen, angemessen vergüten und Arbeitsbedingungen verbessern: Die professionelle Pflege leistet eine wesentliche fachliche und gesellschaftliche Aufgabe. Die Rahmenbedingungen für die professionelle Pflege entsprechen dieser Bedeutung nicht. Die Mehrzahl der Beschäftigten in der Pflege sind Frauen. Die Bewertung ihrer Arbeit ist daher auch unter gleichstellungspolitischen Aspekten zu verbessern.

\section{Der djb fordert:}

- Die Arbeitsbedingungen für Erwerbstätige in der Pflege auch in Privathaushalten - müssen eine langfristige und zufriedenstellende, gesundheitserhaltende Beschäftigung ermöglichen. Dazu gehören entsprechende Arbeitsschutzgesetze, auch bei Beschäftigung im Privathaushalt, und eine angemessene Vergütung. 


\section{Kommission Öffentliches Recht, Europa- und Völkerrecht'}

\section{Fachgruppe Migrantinnen}

Die Gewährleistung von rechtlicher und sozialer Teilhabe von Menschen mit Migrationshintergrund muss auch im Jahr 2013 das Ziel nachhaltiger, die Integration dieser Menschen fördernder Politik sein.

Für eine gelungene Integrationspolitik unter menschenrechtlichen aber auch unter demografischen Gesichtspunkten sind gesicherte Aufenthalts- und Teilhaberechte wesentlich.

Darüber hinaus kann eine effektive und ernst gemeinte Bekämpfung des Menschenhandels und der in unmittelbarem Zusammenhang damit begangenen schweren Straftaten nur Hand in Hand mit gezielten und nachhaltigen Maßnahmen zum Schutz, zur Unterstützung und zum Beistand der Opfer erreicht werden, wobei begleitete und unbegleitete Minderjährige als ganz besonders schutzbedürftig anzusehen sind.

\section{Doppelte Staatsbürgerschaft}

Mit Beginn des Jahres 2013 werden die ersten optionspflichtigen jungen Menschen 23 Jahre alt und müssen daher spätestens an ihrem Geburtstag von ihrem kraft Gesetzes bestehendem Wahlrecht Gebrauch machen. In vielen Fällen ist den Optionspflichtigen diese Konsequenz nicht klar. Während im Jahr 20133.300 junge Deutsche das Optionsverfahren durchlaufen müssen, steigt diese Zahl ab dem Jahr 2018 auf zum Teil deutlich über 40.000 Personen pro Jahr an.

Der automatische Verlust der deutschen Staatsbürgerschaft bei nicht frist- und formgerechter Ausführung des Wahlrechts führt zu unverhältnismäßigen sozialen und rechtlichen Härten.

Diese Härten ergeben sich zum einen daraus, dass die betroffenen Personen seit Jahren deutsche Staatsbürger sind, sich integriert haben und nun vor die Wahl gestellt werden, entweder die deutsche Staatsangehörigkeit, mit der sie groß geworden sind, abzugeben, oder aber diejenige ihrer Eltern. Zum anderen ergeben sich nicht zu rechtfertigende Ungleichbehandlungen und damit Härten im Vergleich zu denjenigen Personengruppen, bei denen eine doppelte Staatsangehörigkeit hingenommen wird. Dies gilt insbesondere deshalb, weil $\mathbb{\$} 29$ Absatz 4 StAG die Möglichkeit, eine Genehmigung zur Beibehaltung der anderen Staatsangehörigkeit zu erhalten, ausdrücklich vorsieht.

\section{Der djb fordert,}

- die Optionspflicht abzuschaffen.

\section{Opferschutzrichtlinie}

Die Richtlinie 2011/36/EU des Europäischen Parlaments und des Rates vom 5. April 2011 zur Verhütung und Bekämpfung des Menschenhandels und zum Schutz seiner Opfer legt spezifische Maßnahmen zum Opferschutz fest. Mit dem Entwurf des BMJ zur Umsetzung der genannten Richtlinie werden nur einzelne, insbesondere strafrechtliche Teile der Richtlinie umgesetzt und nur sehr beschränkte Aufenthaltsrechte für die Betroffenen - meist sind dies Frauen - gewährt. Eine effektive und ernst gemeinte Bekämpfung des Menschenhandels und der in diesem Zusammenhang begangenen schweren Straftaten kann nur Hand in Hand mit gezielten und nachhaltigen Maßnahmen zum Schutz der Opfer sowie zu deren Unterstützung und Beistand erreicht werden. Unbegleitete Kinder und Minderjährige sind als ganz besonders schutzbedürftig anzusehen.

Erwägungsgründe und Regelungen der Richtlinie 2011/36/ EU verlangen eine Berücksichtigung der Situation der Opfer sowie die Prävention, den Schutz vor Menschenhandel, die Beachtung der besonderen Schutzwürdigkeit von Kindern und unbegleiteten Minderjährigen, spezifisch erforderliche Schutzmaßnahmen und Betreuungsangebote, den Zugang zur Rechtsberatung sowie den Einsatz nationaler Berichterstatter. Dies alles bleibt derzeit unbeachtet.

\section{Der djb fordert}

- Schutzsysteme, bei denen betroffenen Frauen über das bloße Bleiberecht zur Durchführung des Strafverfahrens hinaus Aufenthaltsrechte zuerkannt werden. Diese Frauen müssen außerdem staatliche Integrations-, Schutz- und Leistungssysteme in Anspruch nehmen können, die gewährleisten, dass sie sich aus den gewaltbehafteten Verhältnissen lösen können.

\section{Asylbewerberleistungsgesetz}

Das Bundesverfassungsgericht hat in seiner Entscheidung vom 18. Juli 2012 festgestellt, dass die Höhe der Geldleistungen nach $\mathbb{\int} 3$ des Asylbewerberleistungsgesetzes evident unzureichend ist, weil diese den sich verändernden Bedingungen seit 1993 bis heute nicht angepasst wurden. Des Weiteren hat es ausgeführt, dass Artikel 1 Absatz 1 GG in Verbindung mit dem Sozialstaatsprinzip des Artikels 20 Absatz 1 GG ein Grundrecht auf Gewährleistung eines menschenwürdigen Existenzminimums garantiert und dass Artikel 1 Absatz 1 GG diesen Anspruch als Menschenrecht begründet. Er umfasst sowohl die physische Existenz des Menschen als auch die Sicherung der Möglichkeit zur Pflege zwischenmenschlicher Beziehungen und ein Mindestmaß an Teilhabe am gesellschaftlichen, kulturellen und politischen Leben. Das Grundrecht steht deutschen und ausländischen Staatsangehörigen, die sich in der Bundesrepublik Deutschland aufhalten, gleichermaßen zu.

Der Gesetzgeber ist danach verpflichtet, umgehend eine Neuregelung von sozialen Leistungen für den bisher vom Asylbewerberg+esetz umfassten Personenkreis zu schaffen.

\section{Der djb fordert}

- die Anpassung der sozialen Leistungen für Asylsuchende und Personen, die um subsidiären Schutz nachsuchen, an die Leistungen nach dem SGB II und SGB XII ohne Minderung und damit

- die Abschaffung des Asylbewerberleistungsgesetzes.

1 Redaktion: Sabine Overkämping und Zümrüt Turan-Schnieders, Kommission Öffentliches Recht, Europa- und Völkerrecht. 


\section{Fachgruppe Öffentliches Dienstrecht}

Eine gleichberechtigte Teilhabe von Frauen im öffentlichen Dienst ist auch im Jahr 2013 noch nicht erreicht.

\section{Beurteilungen}

Beförderungen hängen im öffentlichen Dienst in erster Linie von Beurteilungen ab. Obwohl die Frauen am Anfang ihres Berufslebens im Vergleich zu den Männern mit besseren Beurteilungen starten, kehrt sich dieses Verhältnis im Laufe ihres Berufslebens um. Familienbedingte Unterbrechungen oder Reduzierungen sind bei Frauen immer noch häufiger und führen manchmal zu Altersproblemen beim Laufbahnwechsel. Sie führen aber auch zu Ausfallzeiten in den Beurteilungen.

Da Beurteilte in der Regel von Beurteilung zu Beurteilung besser werden, könnten und müssen diese Benachteiligungen in begrenztem Umfang durch fiktive Fortschreibungen der Beurteilungen (zu den Grenzen: vgl. BVerwG, Urteil vom 16. Dezember 2010 - BVerwG 2 C 11.09 -) aufgehoben werden. Es genügt nicht, wenn dies nur anlässlich eines Stellenbesetzungsverfahrens geschieht (vgl. $\mathbb{S} 33$ Abs. 3 BLV).

\section{Der djb fordert,}

- eine regelmäßige Nachzeichnung bei Ausfallzeiten, die auf Gründe zurückzuführen sind, die nicht zum Anlass für eine Diskriminierung genommen werden dürfen (z.B. familienund pflegebedingte Ausfallzeiten), gesetzlich vorzusehen.

Frauen sind in den Beurteilungen in den beförderungsrelevanten Spitzennoten nicht entsprechend ihres Anteils in den jeweiligen gleichen Statusämtern vertreten; Teilzeitbeschäftigte werden in der Regel schlechter beurteilt (vgl. zu beidem BT-Drs. 17/4307, insbes. S. 218 ff.). Dies ist auf beiden Seiten (Beurteilte/r und Beurteiler/in) vielfach nicht bekannt. Hier hilft eine regelmäßige Evaluation der Beurteilungen und ein Öffentlichmachen der Beurteilungsergebnisse, soweit dies mit dem Personaldatenschutz vereinbar ist. Beurteilungen sind durch vielfache Zielvorgaben vorgeprägt, so dass eine Einführung von Geschlechterquoten als weitere Zielvorgabe gefordert werden muss (s. auch die noch immer hochaktuelle Stellungnahme des djb vom 15. Februar 2005, zugleich mit näheren Einzelheiten, u.a. Geschlechterquoten in den Beurteilungen, Evaluierung von Beurteilungen).

\section{Der djb fordert daher,}

- dass mit der regelmäßigen Auswertung der Beurteilungsergebnisse nach dem Anteil der Frauen und Männer und der Teilzeitbeschäftigten in den jeweiligen Notenstufen auch die Gründe von Unterrepräsentanzen evaluiert werden.

\section{Gleichstellungspläne und aktive Frauenförderung}

Um sich für Führungspositionen zu qualifizieren, müssen Beschäftigte vielfach zusätzliche Sonderverwendungen vorweisen. Damit auch Frauen hierzu gleichen Zugang haben, fordert der djb, dass diese
- ausgeschrieben werden (Interessenbekundungsverfahren) und

- auch für Teilzeitbeschäftigte zugänglich sind.

\section{Weiter fordert der djb, dass}

- Frauen aktiv angesprochen und gefördert werden sowie

- familienfreundliche, wie z.B. ortsnahe „Erprobungs-“ oder Qualifikationsalternativen, angeboten werden.

Gleichstellungspläne sind mit einem frauenfördernden Personalentwicklungskonzept zu verbinden. Geschlechtsspezifisch nach Ämtern und Beschäftigungen aufgefächerte Strukturdaten für den öffentlichen Dienst einschließlich der Eigenbetriebe in öffentlicher Hand (z.B. Bahn und Post) sind jährlich zu veröffentlichen. Sie müssen auch entsprechend über die Neubesetzung von Stellen in diesem Zeitraum Auskunft geben.

\section{Mitbestimmung/Personalvertretung}

Wie im Betriebsverfassungsgesetz (vgl. dort $\mathbb{} 15$ Abs. 2 BetrVG und $\mathbb{S} 55$ und 15 der Wahlordnung) ist auch im BundesPersonalvertretungsgesetz eine zwingende Verpflichtung zur der Beschäftigtenquote angemessenen Vertretung von Frauen in den Mitbestimmungsgremien vorzusehen.

\section{Der djb fordert,}

- die seit 2001 für die Privatwirtschaft geltenden Regelungen im Betriebsverfassungsgesetz und in der dazugehörigen Wahlordnung zur angemessenen Berücksichtigung des Geschlechterverhältnisses auf den öffentlichen Dienst zu übertragen.

\section{Krankenkosten/Krankenversicherungsschutz für Teilzeit- beschäftigte}

Teilzeitbeschäftigte Beamtinnen und Beamte müssen, um den gleichen Gesundheitsschutz wie Vollzeitbeschäftigte zu erlangen, gleich hohe Beiträge für ihre private Krankenversicherung zahlen wie Vollzeitbeschäftigte. Damit erhalten teilzeitbeschäftigte Beamtinnen und Beamte in Relation zu vollzeitbeschäftigten Beamtinnen und Beamten netto einen geringeren Stundenlohn bzw. eine geringere Nettoalimentation. Um dies aufzufangen, müsste - wie bei Versorgungsberechtigten (vgl. \46 Abs. 2 Nr. 2 BBhV) und Beihilfeberechtigten mit mindestens zwei berücksichtigungsfähigen Kindern (vgl. $\mathbb{S} 46$ Abs. 3 Satz $1 \mathrm{BBhV)}$ - der Beihilfebemessungssatz entsprechend erhöht werden.

\section{Der djb fordert}

- für teilzeitbeschäftigte Beamtinnen und Beamte eine adäquate Erhöhung der Beihilfebemessungssätze, damit die in Relation höheren Kosten der privaten Krankenversicherung aufgefangen werden können.

\section{Fachgruppe Europa}

Auf europäischer Ebene ist eine gleichberechtigte Teilhabe von Frauen seit Langem ein Thema. Immer wieder sind entschei- 
dende geschlechtergleichstellungspolitische Aktivitäten von Europa - den Mitgliedstaaten und den EP-Abgeordneten auf Vorschlag der Europäischen Kommission (jüngst: überarbeitete Beschäftigungsrichtlinie 2006/54/EG und Selbständigenrichtlinie 2010/41/EU) oder den Europäischen Sozialpartnern (überarbeitete Elternzeitrichtlinie 2010/18/EU) - angestoßen worden. Trotz einschränkender Gesetzgebungskompetenzen (Art. 19, 157 Abs. 3 AEUV) gibt es auch 2013 und darüber hinaus Handlungsbedarf, der von der deutschen Politik bislang blockiert wurde.

\section{Richtlinie Frauenquote}

Die Europäische Kommission hat im November 2012 einen Vorschlag zur Einführung einer europaweiten Frauenquote für Aufsichtsräte in großen börsennotierten Unternehmen vorgelegt. Deutschland blockiert diesen Vorschlag auf EU-Ebene. Der djb hält es für notwendig, dass es diesbezüglich europaweit geltende Standards gibt mit Blick auf Artikel 23 GRC und Artikel 157 Absatz 4 AEUV.

\section{Der djb fordert:}

- Deutschland soll sich in den Verhandlungen auf EU-Ebene für eine europaweit geltende Quotenregelung einsetzen.

\section{Antidiskriminierungsrichtlinie}

Die Europäische Kommission hat bereits im Juli 2008 einen Richtlinienvorschlag zur Bekämpfung der Antidiskriminierung außerhalb der Beschäftigung vorgelegt. Deutschland blockiert diesen Vorschlag auf EU-Ebene. Der djb hält es für notwendig, dass es diesbezüglich europaweite Standards mit Blick auf Artikel 21 GRC und Artikel 19 AEUV gibt. Der djb vermisst zudem die Berücksichtigung des Aspekts Geschlecht.

\section{Der djb fordert:}

- Deutschland soll sich in den Verhandlungen auf EU-Ebene für europaweit geltende Standards der Antidiskriminierung außerhalb der Beschäftigung einschließlich des Aspekts Geschlecht einsetzen und auf eine zügige Verabschiedung hinwirken.

\section{3. Überarbeitung der Mutterschutzrichtlinie}

Die Europäische Kommission hat bereits im Oktober 2008 einen Vorschlag zur Überarbeitung der Mutterschutzrichtlinie vorgelegt. Deutschland blockiert diesen Vorschlag auf EUEbene. Der djb begrüßt die lang erwartete Weiterentwicklung der Mutterschutzregelungen und ist überzeugt, dass weitergehende europaweite Standards notwendig sind.

\section{Der djb fordert:}

- Deutschland soll sich in den Verhandlungen auf EU-Ebene konstruktiv einbringen und für eine zügige Verabschiedung einsetzen.

\section{Europäische Staatsanwaltschaft}

Derzeit arbeitet die Europäische Kommission an einem Vorschlag zur Einrichtung einer Europäischen Staatsanwaltschaft zur Wahrung der finanziellen Interessen der Union. Der djb hält die Einrichtung für richtig und ist überzeugt, dass der Europäischen Staatsanwaltschaft weitere Aufgaben übertragen werden sollten.

\section{Der djb fordert :}

- Deutschland soll sich für die zügige Einrichtung der Europäischen Staatsanwaltschaft einsetzen und

- eine gemäß Artikel 86 Absatz 4 AEUV mögliche Ausweitung der Kompetenzen auf den Menschenhandel anregen.

\section{Europawahl 2014}

Für viele Bürgerinnen und Bürger - auch in Deutschland ist Europa ein rotes Tuch. Die europäische Gesetzgebung hat gleichzeitig einen großen Einfluss auf die nationalen Gesetzgebungen. Das ist unabdingbar, da ein gemeinsamer, 28 Mitgliedstaaten umspannender Rahmen ein vernünftiges und geordnetes Miteinander ermöglicht. Europa ist keine Zwangsjacke, sondern bietet uns neben dem uns mittlerweile selbstverständlichen Euro und der Freizügigkeit Chancen und Perspektiven, die wir national nicht verwirklichen können.

Die Bewältigung der (Finanz-)Krise in Europa liegt allen am Herzen. Deren zügige Erledigung erfordert neben neuen fiskalpolitischen Instrumenten auf europäischer Ebene insbesondere die Nutzung bereits vorhandener, wie z.B. die Inanspruchnahme des Europäischen Arbeitsamts EURES vor allem zur Überwindung der Jugendarbeitslosigkeit. Auch für die Bewältigung des Fachkräftemangels ist ein europäischer Ansatz unverzichtbar.

Mit der in 2014 bevorstehenden Wahl zum Europäischen Parlament und der Neubesetzung der Europäischen Kommission soll diese Herausforderung angenommen werden. Ein direkt gewähltes Parlament hat die notwendige Legitimation, die Interessen der Bürgerinnen und Bürger auf Augenhöhe mit den im Rat versammelten Vertreterinnen und Vertretern der Mitgliedstaaten im Gesetzgebungsprozess zu verhandeln. Eine unabhängige Europäische Kommission, die europäische Gesetzgebungsprozesse einleitet mit dem Ziel der Fortentwicklung Europas, garantiert eine genaue Analyse und Aufbereitung von zu lösenden Problemen und damit eine Überwindung von Partikularinteressen oder ausschließlich mitgliedstaatlich fokussierten Belangen.

Gegenwärtig sind nur 35 Prozent der Mitglieder des Europäischen Parlaments Frauen. Bislang hat es nur zwei Präsidentinnen des Europäischen Parlaments gegeben. Nach der Halbzeitwahl 2012 gibt es nur noch drei Vizepräsidentinnen neben elf Vizepräsidenten (ein Rückgang von 43 auf $21 \%)$.

Das Kollegium der Europäischen Kommission setzt sich derzeit aus neun Kommissarinnen und 18 Kommissaren zusammen. Auch diese Zusammensetzung ist unter geschlechterparitätischen Gesichtspunkten verbesserungsfähig.

Gleiche Repräsentation von Frauen und Männern in der öffentlichen Meinungsbildung und Entscheidungsfindung 
ist ein Grundrecht (Art. 23 GRC mit weiterer primärrechtlicher Ausformung in Art. 3 Abs. 3 UAbs. 2 Satz 1 EUV, Art. 8 AEUV). Die Unterrepräsentation von Frauen stellt ein gravierendes Demokratiedefizit dar, das der demokratischen Ausrichtung der Europäischen Union abträglich ist. Demokratie braucht Geschlechtergerechtigkeit!

Die Kandidatinnen und Kandidaten für das Europäische Parlament und die Europäische Kommission müssen über europapolitische Kompetenz verfügen. Selbstverständlich ist auch ihre Unabhängigkeit von nationalen Regierungen. Nur so kann eine erfolgversprechende Vision für das Europa der Zukunft entstehen und können die notwendigen Reformen innerhalb und außerhalb der Institutionen durchgeführt werden. Eine geschlechterparitätisch besetzte Europäische Kommission und ein geschlechtergerechtes Europäisches Parlament sind hierfür Voraussetzung.

\section{Der djb fordert:}

- Die deutschen Parteien sollen ihren Beitrag zu einer geschlechtergerechten Zusammensetzung des Europäischen Parlaments leisten, indem sie ihre Listen für die Europawahlen geschlechterparitätisch aufstellen.

- Deutschland soll jeweils eine Kandidatin und einen Kandidaten für das Kollegium der Europäischen Kommission sowie für alle Top-Jobs in den Institutionen der Europäischen Union benennen.

- Deutschland soll auch darauf dringen, dass eine Präsidentschaftskandidatin sowie ein Präsidentschaftskandidat für das Europäische Parlament nominiert werden. Dies gilt auch für die Nominierung der Vorsitzenden der Ausschüsse des Europäischen Parlaments und der Büros.

\section{Fachgruppe Völkerrecht}

Frauenrechte sind ein wichtiger Bestandteil des universellen Völkerrechts. Insbesondere das UN-Übereinkommen über die Beseitigung jeder Form der Diskriminierung der Frau (CEDAW) kodifiziert die gleichen Menschenrechte von Frauen umfassend und verdeutlicht eine aktive Pflicht der Nationalstaaten, auf allen Gebieten alle geeigneten Maßnahmen zu ergreifen, um eine tatsächliche gleichberechtigte Teilhabe von Frauen zu erreichen. Die im Völkerrecht verwurzelten Frauenrechte spielen in der deutschen Politik, Rechtsprechungspraxis und im öffentlichen Bewusstsein keine angemessene Rolle. Sie entfalten ihre Potentiale in Deutschland nur unzureichend.

Eine menschenrechtsbasierte Analyse von Politikfeldern und Strategien würde es ermöglichen, die Ursachen von Diskriminierungen von Frauen genau zu benennen und die nationale Politik entsprechend zieldifferenzierter auszurichten. Hierfür wäre es unter anderem wichtig, beim Abfassen der deutschen Staatenberichte an die Menschenrechtsausschüsse alle Bundesministerien und Landesregierungen besser einzubeziehen und die Zivilbevölkerung angemessen zu beteiligen und zu informieren. Der CEDAW-Ausschuss hat sich in seinen Verhandlungen mit der deutschen Regierung sehr kenntnisreich mit spezifischen deutschen Defiziten in der Gleichstellungspolitik beschäftigt. Die Anregungen des Ausschusses wurden in der deutschen Politik aber bisher nicht ausreichend beachtet.

\section{Der djb fordert daher}

- eine menschenrechtsbasierte Analyse von Politikfeldern und Strategien und eine bewusstere Auseinandersetzung aller politisch Agierenden mit den abschließenden Bemerkungen und den Allgemeinen Empfehlungen des CEDAWSachverständigenausschusses.

\section{„Rote Roben“- Bundesrichterwahlen'}

1. Ein klares Zeichen für den Willen der künftigen Bundesregierung, das Gleichstellungsdefizit für (potentielle) Kandidatinnen bei den Richterwahlen zu beseitigen, wäre die Novellierung des Richterwahlgesetzes. Mit seinen Formulierungen „der Vorgeschlagene“ und „der Gewählte“ gibt es vor, die Wahl von männlichen Kollegen sei der Normalfall - was es bislang fast immer war.

2. Die Gleichberechtigung von Frauen und Männern bei den Bundesrichterwahlen durchzusetzen, muss konsequenter verfolgt werden. Ein Anteil von Richterinnen an den obersten Bundesgerichten von nur 20 bis 30 Prozent, der jährlich nur marginal wächst, ist nicht akzeptabel. Deshalb müssen künftig bei jeder Wahl mindestens 50 Prozent Frauen für jedes Bundesgericht gewählt werden.

3. Dieses Ziel durchzusetzen helfen die Gleichstellungsbeauftragten der obersten Bundesgerichte. Ihnen ist ein Teilnahme- und Beratungsrecht in Sitzungen des Präsidialrats zu geben entsprechend der Schwerbehindertenvertretung ( $\int 95$ Abs. 4 SGB X).
4. Eine paritätische Besetzung des Richterwahlausschusses bei den Mitgliedern kraft Wahl würde die Absicht der im Bundestag vertretenen Parteien dokumentieren, dass sie es mit der Durchsetzung der Gleichstellung von Bundesrichterinnen und Bundesrichtern im Sinn des Artikels 3 Absatz 2 Satz 2 GG ernst meinen.

1 Eva Schübel, Vizepräsidentin des djb. 